\title{
DE LA CASA AL BARRIO
}

MARGARITA GREENE

FELIPE LINK

RODRIGO MORA CRISTHIAN FIGUEROA

PROFESORA E INVESTIGADORA DEL CENTRO DE DESARROLLO SUSTENTABLE, PONTIFICIA UNIVERSIDAD CATÓLICA DE CHILE, CHILE.

PROFESOR E INVESTIGADOR DEL CENTRO DE DESARROLLO SUSTENTABLE, PONTIFICIA UNIVERSIDAD CATÓLICA DE CHILE, CHILE. PROFESOR, UNIVERSIDAD DIEGO PORTALES, CHILE.

COLABORADOR E INVESTIGADOR DEL LABORATORIO DE CIUDAD Y MOVILIDAD FADEU, PONTIFICIA UNIVERSIDAD CATÓLICA DE CHILE, CHILE.

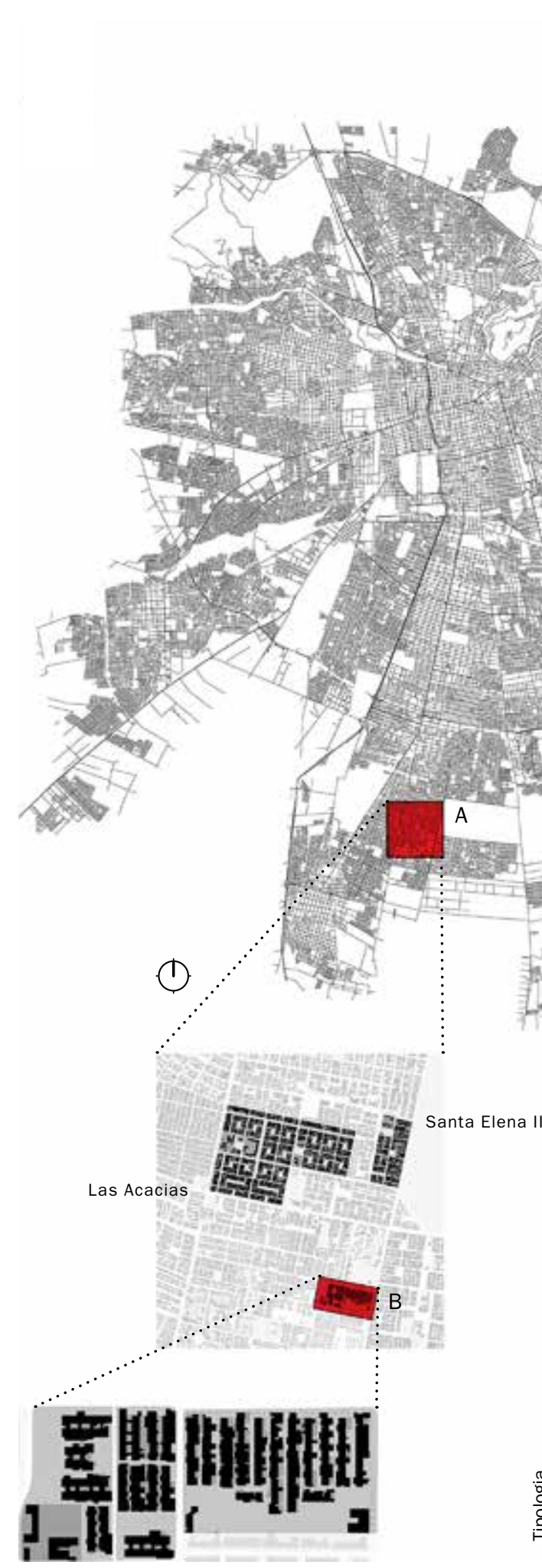

FIG 1

Ubicación y grano edificatorio de los conjuntos analizados/ Location and typology of the analyzed complexes.

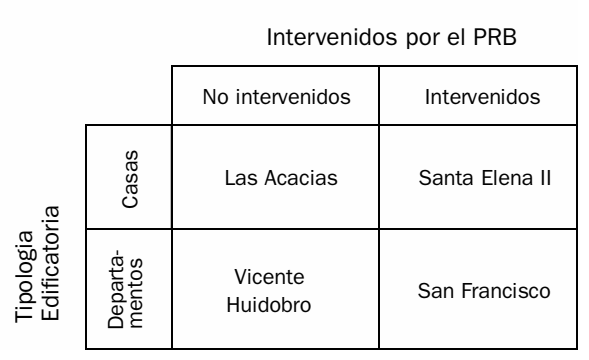

CUADRO 1

Muestra de barrios analizados/ Sample of analyzed neighborhoods.

UNA MIRADA SOCIO-ESPACIAL: DE MÁS VIVIENDAS A MEJORES BARRIOS

uㅔ $n$ más de 100 años de historia la política habitaInII cional chilena se ha ido adaptando continuamente a la coyuntura social, económica y política del país, adoptando estrategias y soluciones diversas según el momento. Así pasó de un enfoque de Estado proveedor a uno subsidiario, de entregar grandes conjuntos habitacionales de viviendas terminadas, a soluciones habitacionales progresivas de menor escala que debían ser terminadas por los propios pobladores. Se pasó desde un modelo basado principalmente en el financiamiento público, a uno donde el sector privado es el protagonista. Asimismo, en los últimos años tanto autoridades como expertos han manifestado la necesidad de un nuevo cambio de mirada a la política habitacional. Este cambio apunta principalmente a incorporar una "mirada barrial" a la política habitacional, mejorando tanto las viviendas como su entorno.

La racionalidad que sustenta este último cambio parece tener sus raíces, al menos teóricas, en dos textos de los últimos 10 años: Nueva pobreza urbana de Manuel Tironi -escrito en 2003-y Los con techo de Alfredo Rodríguez y Ana Sugranyes, publicado en 2005. Aunque diferentes en su enfoque, el primero se centra en las dimensiones sociales y ambientales de lo que significa actualmente ser pobre en Chile. La segunda obra se enfoca en la ausencia de comunidad en los conjuntos de vivienda social construidos en Chile desde los años noventa. Ambos coinciden en que los problemas de las ciudades chilenas en la actualidad ya no se asocian a carencias materiales o alimentarias básicas -como la existencia de un techo o la desnutrición- sino más bien a temas de vulnerabilidad y exclusión social (Wacquant, 2001).

El diagnóstico anterior se enmarca en un momento en que, después de más de 30 años de políticas habitacionales con énfasis en la producción de nuevas soluciones, por primera vez se vislumbra la superación del déficit habitacional cuantitativo en el corto plazo (Hidalgo, 2007). Esta realidad ha hecho posible repensar el diseño de las políticas e instrumentos habitacionales tradicionales centrados en la producción de soluciones habitacionales por sobre el entorno urbano donde estas se instalan. Así, el mejoramiento del espacio público, de las relaciones entre vecinos $\mathrm{y}$ de la participación ciudadana, adquieren cada vez más relevancia en el discurso sectorial. Ya no se trata solo de producir viviendas, sino de que tanto las nuevas como las existentes formen parte de barrios con equipamiento y servicios que, a su vez, estén adecua- 
Como componente estructural de la realidad urbana, la calle suele tomarse como un hecho dado y hasta cierto punto, obvio. La reflexión sobre las primeras normativas que regularon su naturaleza da pistas sobre los destinos y roles que, en nuestra realidad contemporánea, la calle puede asumir.

PALABRAS CLAVE URBANISMO - CHILE, VIVIENDA SOCIAL, SEGREGACIÓN, COMUNIDAD, ESPACIO PÚBLICO.

damente insertos en sus ciudades. En resumen, se cambia el enfoque desde una perspectiva cuantitativa a una cualitativa y desde una visión "sectorial", centrada en lo estrictamente habitacional, a una más integral que incluye el mejoramiento de la infraestructura y del barrio, además del fortalecimiento de la comunidad.

\section{EL BARRIO COMO UNIDAD DE ANÁLISIS}

Este nuevo paradigma ha significado el surgimiento del barrio como unidad de intervención urbana. Los valores del barrio como una unidad territorial identificable han sido promovidos desde mediados del siglo pasado. Hacia 1960 Lynch hacía hincapié en sus características morfológicas, mientras que Jacobs enfatizaba sus valores por su capacidad de permitir que sus habitantes se pudieran reconocer e intercambiar información. El concepto de barrio para Lefevre (1967) aparece como el punto de contacto más accesible entre el espacio geométrico y el espacio social, es decir, como el elemento que articula el espacio social con el espacio físico y cuya estructura depende en gran medida de la estructura general de la ciudad.

Para Mayol, en el artículo "El barrio" de 2006, este se erige en prácticas como el saludar, caminar de determinada manera o el conocimiento de unos a otros en una relación de vecindad en la que forjan poco a poco una identidad común, generadora de confianza y pertenencia. Entonces el barrio sería el resultado de un imaginario colectivo en tanto que reúne las ideas de comunidad de los habitantes, operando como una antesala al hogar. El barrio también es el lugar donde el espacio es negociado, donde los diferentes grupos que lo habitan intentan, a veces soterradamente, a veces en forma más explícita, imponer sus valores y formas de vida (Gravano, 2003).

De hecho, varios autores identifican la erosión de las relaciones comunitarias como producto de la nueva pobreza urbana. Katzman (2001) sugiere que el proceso de consolidación de barrios vulnerables en Santiago parece limitar el potencial atribuido a la escala barrial, convirtiéndola muchas veces en un elemento más de aislamiento social de los pobres urbanos. Así, esta investigación ${ }^{1}$ intenta avanzar en la comprensión de los procesos de consolidación de barrios y comunidades populares con el objetivo de dar cuenta de la complejidad del barrio como unidad de análisis. Para ello este estudio se realiza a través de una perspectiva sistémica considerando las múltiples variables que interactúan, simultánea y secuencialmente, en más de una escala urbana.
A shifting from quantitative to qualitative issues drives urgent reviews of housing policies in Chile. The mere provision of housing units gives way to the recognition of the neighborhood and its integration into the total city as key factors of new social programs.

KEYWORDS: URBANISM - CHILE, SOCIAL HOUSING, SEGREGATION, COMMUNITY, PUBLIC SPACE. English text in p. 85

\section{LOS CASOS ANALIZADOS}

Partiendo por los barrios del "Programa de Recuperación de Barrios" (PRB) del MINVU del año 2010, se seleccionaron dos conjuntos relativamente cercanos: uno de casas (Santa Elena II) y otro de departamentos (San Francisco) y dos adicionales, uno de cada tipo, que no fueron intervenidos por el PRB (Las Acacias y Vicente Huidobro respectivamente). Los cuatro casos analizados se localizan en la comuna de El Bosque, en la periferia sur de Santiago, entre los ejes de las avenidas Santa Rosa y Gran Avenida. Ubicados dentro de un radio de no más de $500 \mathrm{~m}$, todos están localizados en una de las zonas más pobres de la capital, con escasas áreas verdes y espacios públicos e infraestructura deficitaria (Reyes y Figueroa, 2010). La lógica de la muestra puede ser esquematizada y la ubicación y grano del conjunto pueden ser indicadas sencillamente (cuadro 1 y fig. 1).

La morfología y tipología edificatoria es quizás la variable más evidente -y, por lo tanto, una puerta de entrada casi obvia- a la hora de definir y comprender este tipo de barrios. En este sentido, la selección respondió a aspectos físicos e históricos que dan homogeneidad a un sector de la ciudad: altura de edificación, materialidad predominante, sistema de agrupamiento de las viviendas y data de construcción. Lynch sostiene que la generación de imágenes urbanas coherentes depende, en parte, de la existencia de conjuntos uniformes ya sea por sus características morfológicas como altura, tipología, estilo arquitectónico, o por su historia o estructura urbana (Lynch, 1960).

Hay una serie de autores que sugieren que determinadas tipologías o formas arquitectónicas pueden afectar la calidad de la comunidad. Gehl (2001) plantea que la distancia métrica entre las casas puede influir en el grado de cercanía con los vecinos, al posibilitar una mayor frecuencia de contactos. Por su parte, Newman (1972) sugiere que la clave para la creación de comunidades "sanas" está en la definición de los bordes y límites de los conjuntos. Hillier, en Space is the machine de 1996, postula en cambio que hay aspectos de la trama urbana que pueden, en determinadas circunstancias, generar comunidades enfermas en el sentido de no permitir el acceso de extraños y de disociar los lugares por donde caminan mujeres, hombres y niños. De acuerdo a Hillier, la forma y cantidad en que calles y pasajes se conectan en la ciudad tiene una directa relación con la cantidad de personas que transitan por ellas, lo que crea las condiciones iniciales para que se forjen relaciones entre vecinos. Junto con lo anterior, políticas habita- apoyo del proyecto FONDECYT 1100068 Consolidación de barrios vulnerables desde una perspectiva socioespacial. También cuenta con el apoyo de CEDEUS - Centro de Desarrollo Urbano Sustentable, Chile. 


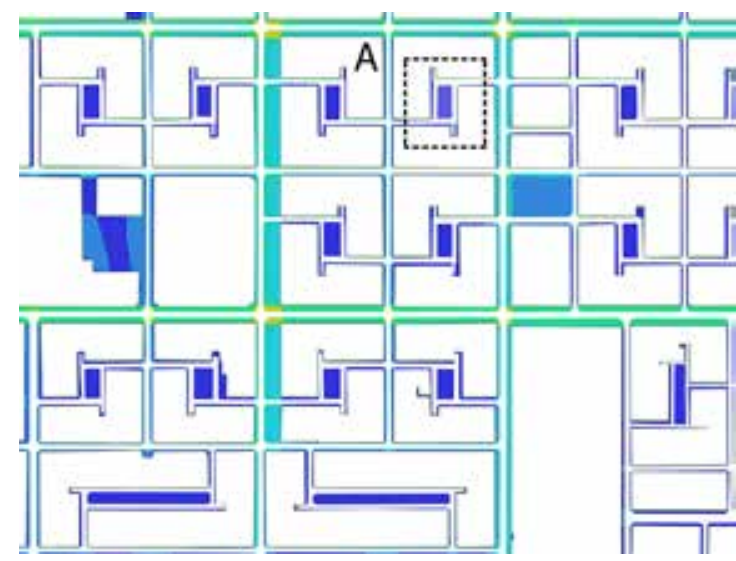

A Las Acacias.

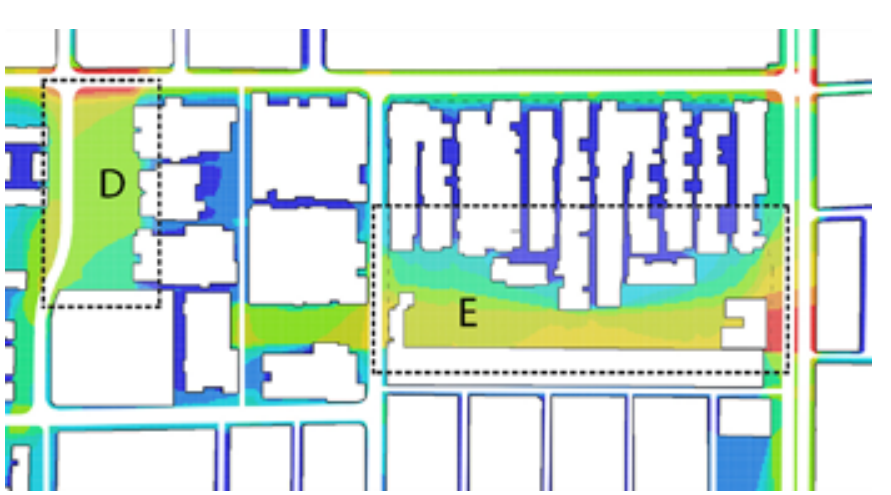

C Vicente Huidobro y San Francisco.

Para efectos del estudio, las plazas se 2 identificaron con letras de la $\mathrm{A}$ a la $\mathrm{E}$.

\section{FIG 3}

Detalle de plazas interiores y puertas de acceso/ Detail of interior plazas and entrances

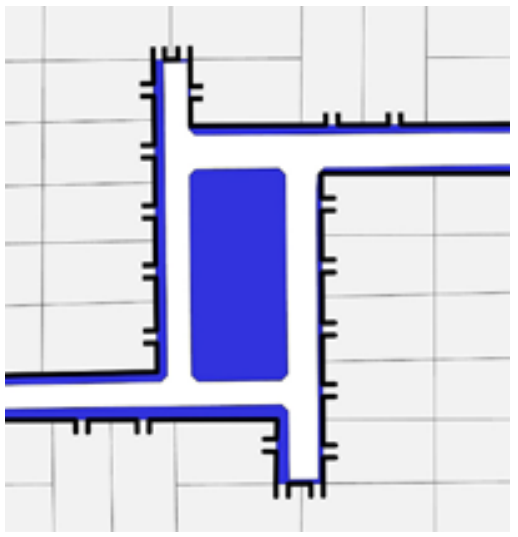

Las Acacias, detalle plaza A. E./S. 1: 1.000

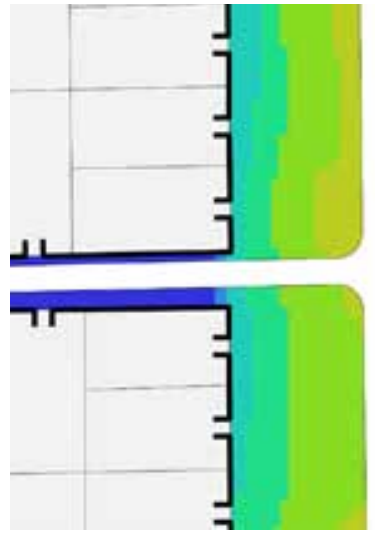

Santa Elena II, detalle plaza B. E./S. 1: 500

느 Accesos a propiedades
CUADRO 2

Esquema comparativo de visibilidad de las plazas de los cuatro conjuntos/ Comparative visibility scheme of the plazas of the four complexes.

\begin{tabular}{|c|c|c|c|}
\hline \multicolumn{2}{|l|}{} & el espacio exterior & interior de las casas \\
\hline Las Acacias & Plazas interiores & - & + \\
\hline Vicente Huidobro & Plaza fondo & + & + \\
\hline San Francisco & Plaza lateral & + & - \\
\hline
\end{tabular}

cionales recientes vinculadas a la seguridad ciudadana, recalcan la importancia de la relación visual entre la casa y la calle, así como entre esta última y la localización de los servicios y equipamientos (Espacios urbanos seguros, 2003).

\section{LA TRIPLE MIRADA SOCIO-ESPACIAL}

Conscientes de que la construcción de lo que se conoce como "espacio barrial" es un fenómeno multidimensional, simultáneamente la investigación abordó el tema desde una perspectiva social y una espacial integrando herramientas y métodos que se complementan y permiten una comprensión más acabada del problema. Para ello se usaron tres enfoques: un análisis de visibilidad espacial de los principales espacios de cada uno de los conjuntos, un estudio de percepción y uso del espacio y, finalmente, un análisis de los límites percibidos del barrio a través de mapas mentales.

\section{ANÁLISIS DE VISIBILIDAD}

El análisis de visibilidad, realizado a través del programa computacional Depthmap, busca dar cuenta de los espacios más y menos visibles del sistema analizado. Para ello se construye una retícula ortogonal de tamaño variable -en este caso de un metro por un metro- sobre el espacio libre de los conjuntos. Luego se miden las relaciones de mutua visibilidad entre todas las celdas resultantes. Con ello se obtiene un valor numérico para cada celda y una visualización en escala de color que va desde los espacios con mayores campos visuales en tonos cálidos, hasta los espacios con menor visibilidad en tonos fríos (fig. 2).

La gráfica, en lo que se refiere a sus espacios públicos, deja ver grandes diferencias de visibilidad entre los cuatro conjuntos, específicamente en relación a las plazas locales² (fig. 2). En primer lugar se destaca que las plazas "A" del conjunto Las Acacias, las que corresponden a plazas interiores a las cuales se accede por pasajes a mitad de manzana, aparecen poco integradas visualmente a su contexto inmediato. Esto quiere decir que son poco visibles desde los otros espacios públicos del barrio. Sin embargo, el trabajo de terreno realizado refleja que estas plazas son controladas visualmente desde las casas que las rodean. Más aún, además de tener ventanas, las viviendas tienen puertas de acceso hacia las plazas. En el caso del conjunto Santa Elena II, los principales espacios públicos, las llamadas plazas "B", se presentan como altamente accesibles desde el punto de vista visual y las casas también tienen ventanas y puertas de acceso hacia las plazas (fig. 3).

En el caso de los edificios, identificados con las plazas "D" y "E", correspondientes a los conjuntos Vicente Huidobro y San Francisco respectivamente, se aprecia que las plazas son más amplias y están más integradas visualmente al espacio público general. Sin embargo, por estar separadas de los edificios de departamentos, no tienen control visual desde el interior ni puertas de acceso que den a estos espacios (cuadro 2).

\section{IDENTIDAD Y SATISFACCIÓN}

Una segunda mirada a los barrios analizados se relaciona con la existencia de prácticas comunes entre 
Evaluación de atributos de la vivienda por barrio/

Assessment of housing attributes according to neighborhood.

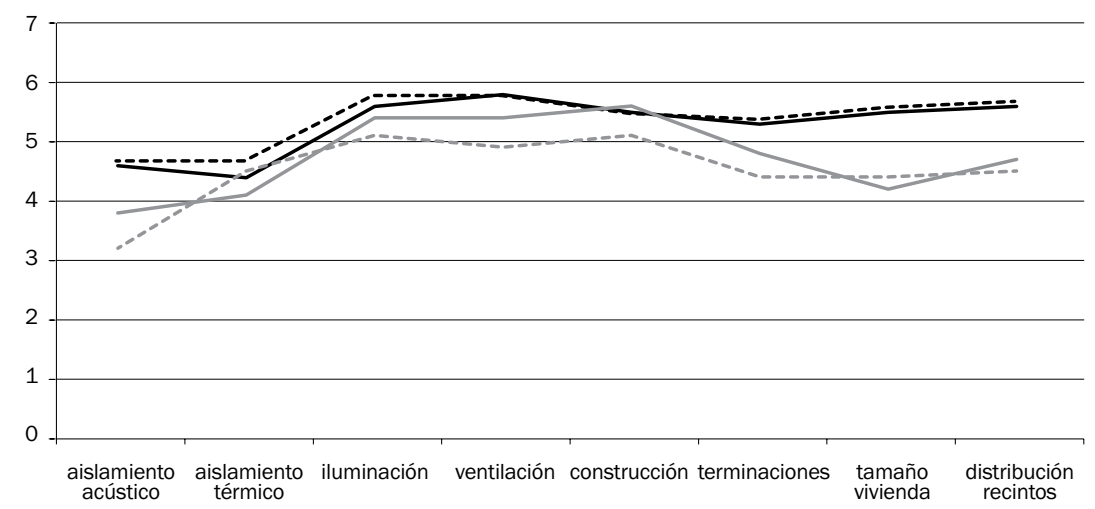

FIG 5

Evaluación de vivienda, comunidad y barrio/

Assessment of housing, community and neighborhood.

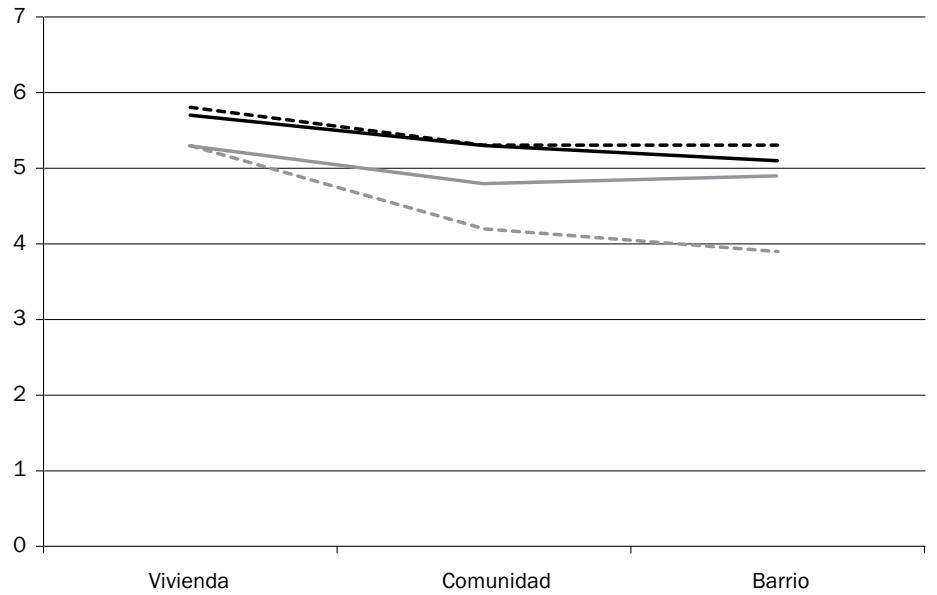

FIG 6

Evaluación de capacidad de vivienda, comunidad y barrio por proveer vida satisfactoria y voluntad de cambio de barrio/ Assessment of housing, community and neighborhood capacity to provide life satisfaction - interest in moving.

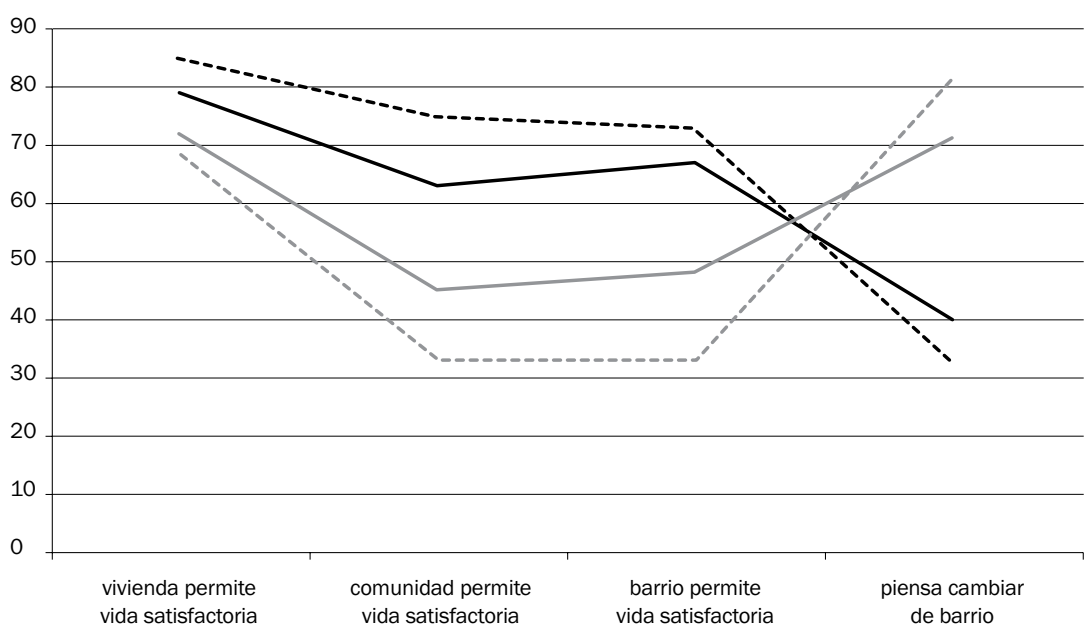

vida satisfactoria vida satisfactoria vida satisfactoria de barrio

-.-.- Casas no intervenidas

Casas intervenidas

Departamentos intervenidos

Departamentos no intervenidos
En las evaluaciones realizadas durante $\mathbf{3}$ este estudio se usó una escala de 1 a 7 , análoga a aquella de las calificaciones escolares en Chile. los habitantes, atendiendo precisamente a la idea del barrio como espacio articulador entre el espacio privado de la vivienda y el espacio urbano generalizado de la gran ciudad. Los resultados de este análisis, a partir de una encuesta presencial realizada a una muestra de 405 habitantes del sector acerca de sus características sociales, arraigo al barrio, satisfacción residencial y patrones de uso del espacio público, arrojaron diferencias evidentes, principalmente, por tipología edificatoria.

Por ejemplo, en relación a las características propias de la vivienda como aislamiento acústico y térmico, iluminación, ventilación, tamaño de la vivienda y otros, se detectó que las casas son mejor evaluadas que los departamentos, independientemente de su intervención por parte de las políticas públicas. En efecto, mientras los conjuntos de casas promediaron en esta materia un puntaje de 5.4 y 5.3 respectivamente $^{3}$, los conjuntos de departamentos promediaron un 4.7 y 4.5 respectivamente. En el gráfico a continuación se observa la variación entre atributos y se aprecia que los departamentos fueron consistentemente peor evaluados que las casas (fig. 4).

De la misma manera, la evaluación de la vivienda como un espacio que permite la realización de actividades domésticas en su interior tales como estudiar, descansar, comer, reunirse, cocinar, bañarse o recibir amigos, es significativamente mejor en los conjuntos de casas que en los de departamentos. Es así como un $45 \%$ de los residentes en los departamentos del conjunto San Francisco y un 49\% de los residentes en el conjunto Vicente Huidobro, declaran que las viviendas no proveen un espacio adecuado de sociabilidad. Estos porcentajes son menores en los conjuntos de casas.

Así, puede observarse una síntesis de la evaluación de la vivienda, barrio y comunidad en los cuatro conjuntos analizados. Puede apreciarse que las mejores calificaciones corresponden a las viviendas y las peores al barrio y que, nuevamente, entre las viviendas los conjuntos de casas son mejor evaluados que los de departamentos. También se aprecia una evaluación significativamente inferior de los departamentos no intervenidos, en comparación a aquellos intervenidos. Esta tendencia se mantiene prácticamente en todos los ámbitos investigados: los conjuntos de casas se mantuvieron en los rangos superiores y los de departamentos en los inferiores. Del mismo modo, se ubican en rango decreciente desde los atributos de la vivienda a los de comunidad y a los de barrio. Los resultados siguieron la misma tendencia cuando se indagó con respecto a la capacidad de la casa, comunidad y barrio acerca de la posibilidad de permitir una vida satisfactoria y si tenían voluntad de mudarse, si ello fuera posible (fig. 5).

Como se observa, se demostró total consistencia entre las malas evaluaciones y la voluntad de cambio (fig. 6). Es importante recordar que los conjuntos San Francisco y Santa Elena II fueron sujetos de intervenciones mayores en sus espacios públicos, específicamente en las escaleras de acceso a los departamentos, en las techumbres de las viviendas y en el equipamiento de plazas. Los datos recogidos señalan que la tipología de casa o departamento fue determinante a 
FIG 7

Mapas cognitivos de los habitantes de los barrios analizados/Cognitive maps by the residents of the neighborhoods analyzed.
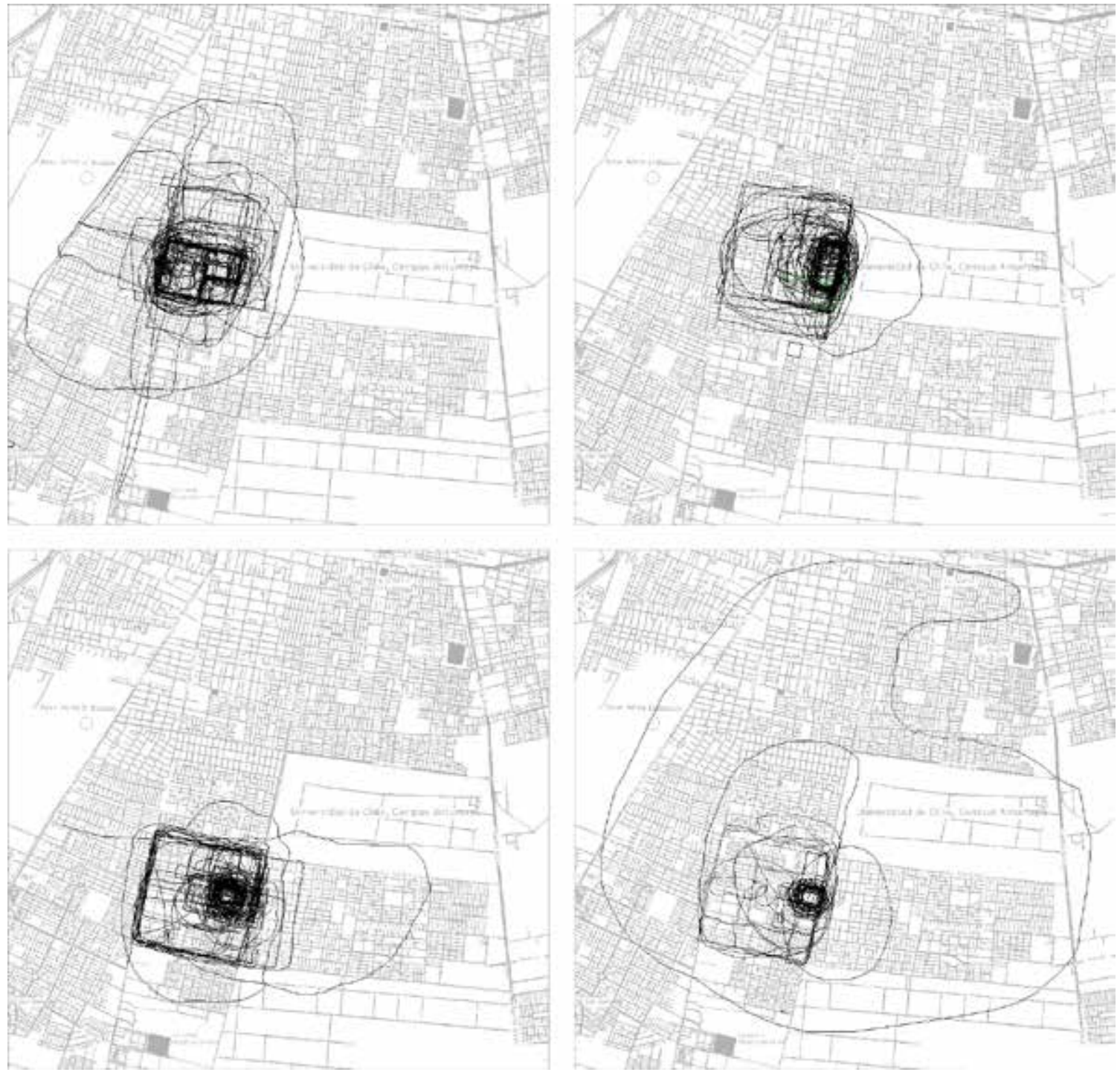

la hora de evaluar, más aún que el haber recibido o no una intervención gubernamental.

Lo anterior sugiere que el impacto de este tipo de programa de intervención es mayor en conjuntos de edificios que en conjuntos de casas y que apunta a mejorar las relaciones entre vecinos y la evaluación con el barrio en general. Estos resultados parecen estar en línea con estudios anteriores que sugieren que, a igual distancia al centro, las personas valorizan mejor las casas que los departamentos (Brain, Iaccobelli y Sabatini, 2005). Dado que los cuatro conjuntos están prácticamente a igual distancia del centro, no es de extrañar que los conjuntos de casas hayan sido mejor evaluados que los de departamentos, incluso después de que uno de estos últimos haya sido intervenido. Por el contrario, la mejoría sustancial del conjunto San Francisco en lo que respecta a arraigo y satisfacción con el barrio, sugiere que las intervenciones del PQB-PRB ${ }^{4}$ han tenido un impacto importante en la calidad de vida de los habitantes.

¿Por qué no ocurrió lo mismo con las casas donde el conjunto no intervenido tuvo mayor valoración que el intervenido? Una posible explicación, factible de ser indagada en el futuro, es que el barrio intervenido efectivamente tenía problemas importantes en su constitución, por lo que las intervenciones podrían haber ayudado a mejorarlo, permitiendo una cercanía con su vecino, mas no alcanzándolo.
Además, dentro de cada barrio se indagó en el efecto de variables de escala local. Así por ejemplo, se encontró que a pesar de la mala evaluación sistemática de los habitantes de los edificios Vicente Huidobro, cuyos departamentos no fueron intervenidos, aparecen algunas diferencias en las evaluaciones subjetivas según la localización específica dentro del barrio y su proximidad con los escasos espacios públicos disponibles. Mientras la nota promedio del barrio por parte de los habitantes de esta población es de un 3.9, la evaluación aumenta a un 4.2 si se vive cerca de alguna plaza y baja a 3.4 si la vivienda se localiza lejos de los espacios públicos. De la misma manera, la nota a la comunidad aumenta de 4.2 a 4.4 si se está cerca de las plazas y baja a 3.7 si se está lejos. Si bien estos resultados no muestran grandes diferencias en los otros barrios analizados, sí se puede inferir alguna influencia de la presencia de plazas en la valoración del barrio. Siguiendo a Borja y Muxi (2003), si bien no se puede pedir al urbanismo que resuelva una serie de problemas que parecen ser de otro orden, por lo menos este no debiera empeorarlos. Por último, se desea resaltar que la evaluación general de la vivienda muestra diferencias menores entre los conjuntos analizados (fig. 5). En este sentido, el paso de la casa al barrio como objeto de intervención pública, adquiere mayor relevancia para la arquitectura y el urbanismo. 


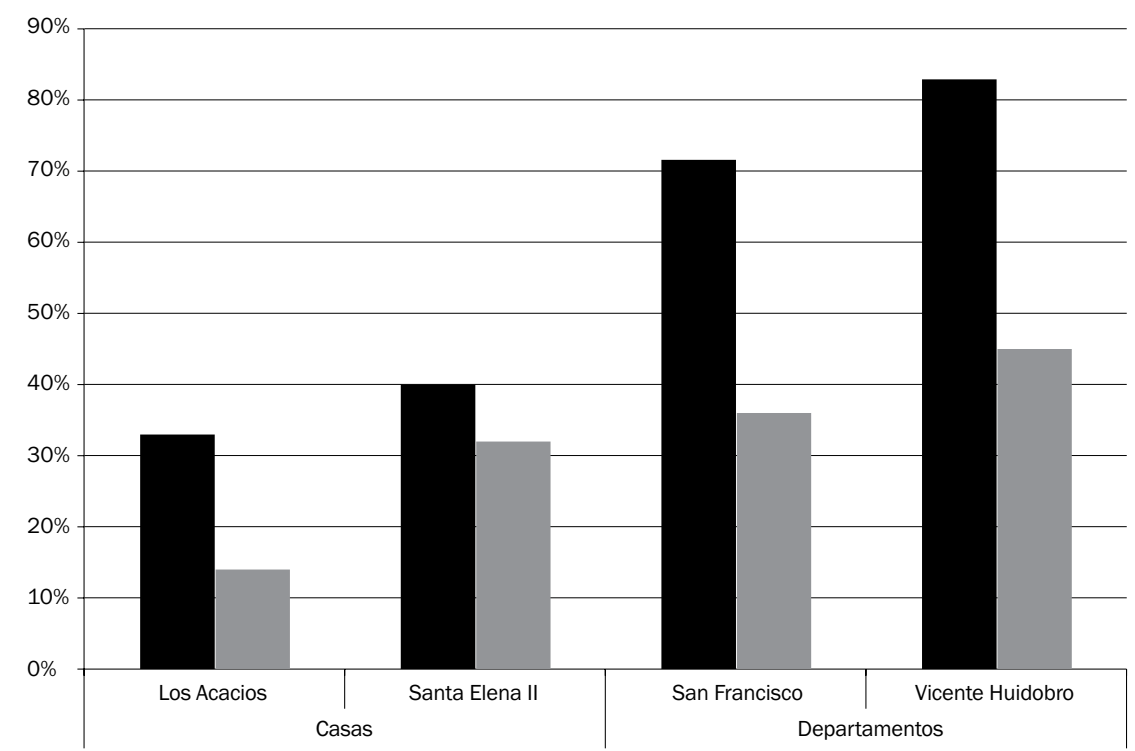

FIG 8

Coincidencia de límites del barrio y voluntad de cambio de barrio. Coinciding limits of the neighborhood and interest in moving.

\begin{tabular}{|r|c|c|c|c|c|}
\cline { 3 - 5 } \multicolumn{2}{c}{} & \multicolumn{2}{c|}{ Área } & \multicolumn{2}{c|}{ Perímetro } \\
\cline { 3 - 6 } & $\begin{array}{c}\text { Promedio } \\
\text { Ha }\end{array}$ & $\begin{array}{c}\text { Desviación } \\
\text { Estándar }\end{array}$ & $\begin{array}{c}\text { Promedio } \\
\text { Ha }\end{array}$ & $\begin{array}{c}\text { Desviación } \\
\text { Estándar }\end{array}$ \\
\hline Casas no Intervenidas & Las Acacias & 532 & 954 & 2524 & 1858 \\
\hline Casas Intervenidas & Santa Elena II & 361 & 504 & 2043 & 1325 \\
\hline Departamentos Intervenidos & San Francisco & 499 & 1085 & 2087 & 1982 \\
\hline Departamentos no Intervenidos & Vicente Huidobro & 507 & 969 & 2235 & 1961 \\
\hline
\end{tabular}

CUADRO 3

Comparación de los mapas cognitivos realizados por los habitantes de los barrios/Comparison of the cognitive maps drawn by the residents.

Nota: Porcentaje de residentes cuyo mapa cognitivo coincide con el del programa de intervención o municipio/Note: The percentage of residents whose cognitive map coincides with the intervention program or municipality.
De este modo, los resultados coinciden con lo planteado por Bourdieu en "El efecto de lugar" de 1999. El proceso de consolidación barrial, analizado desde una perspectiva social, se asocia no solo con las características propias de cada comunidad, sino también con las condiciones físicas de vivienda y entorno como una estructura determinante de relaciones sociales, donde el espacio social se proyecta de alguna manera en el espacio físico.

\section{COGNICIÓN ESPACIAL Y}

\section{REPRESENTACIÓN MENTAL DEL BARRIO}

El tercer enfoque usado en este trabajo se relaciona con la capacidad de los residentes de identificar los límites de lo que consideran su barrio. La mayoría de las aproximaciones a lo barrial, que abordan la delimitación del barrio, se centran en la presencia del "otro" en el espacio, lo que generaría un conflicto por imponer ciertas pautas de comportamiento y valores entre los grupos en disputa de un barrio (Márquez, 2011; Márquez y Pérez, 2008).

El enfoque usado en esta investigación se aparta de esta línea teórica no en el sentido de intentar develar el conflicto entre los diferentes grupos que ocupan el espacio, sino más bien en cómo cada uno establece los límites de lo que consideran como su barrio. Visto así, el enfoque se relaciona con las metodologías tradicionales del tema, como el trabajo de Lynch
(1960) sobre la imagen de los habitantes de Boston de su ciudad. De acuerdo a esta visión, la imagen de una ciudad se originaría a partir del juego entre cinco elementos: nodos, vías, sendas, barrios e hitos, los que, al ser vistos en forma repetida, generarían en el corto plazo lo que se conoce como mapa cognitivo de una ciudad (Tolman, 1948; Siegel y White, 1975; Thorndyke y Hayes-Roth, 1982).

En el presente estudio se pidió a los encuestados que trazaran los límites de lo que consideraban como su barrio. Para ello se les entregó un plano en blanco y negro señalando las calles principales e hitos del sector, lo que dio como resultado distintos mapas cognitivos trazados por los encuestados (fig. 7). En primera instancia se analizaron los mapas calculando los promedios y desviación estándar del área y perímetro de los planos dibujados. Los resultados muestran que de los cuatro conjuntos analizados, tres tienen áreas promedios entre las 499 y las 532 ha, mientras que el conjunto Santa Elena II tiene un área promedio de 361 ha. Además, este último es el que posee menor desviación estándar. Es decir, los dibujos de sus habitantes fueron más similares entre sí que los hechos por los habitantes de los tres conjuntos restantes. A diferencia del área, los perímetros de los dibujos parecen mostrar más homogeneidad: mientras en los conjuntos de casas miden en promedio 2.043 y 2.524, en los de edificios alcanzan 2.087 y $2.285 \mathrm{~m}$ (ver cuadro 3 ).

Por último, se analizó la coincidencia de los bordes trazados por los habitantes con los definidos institucionalmente por el programa de intervención y por la municipalidad. Aquí se encontraron importantes diferencias: los conjuntos de departamentos demostraron más coincidencia que los de casas. En el conjunto Vicente Huidobro fue de un $45 \%$, en el conjunto San Francisco de un $36 \%$, en el conjunto Santa Elena II fue de un $32 \%$ y en el conjunto Las Acacias de un 14 $\%$. Ello parece indicar en primera instancia que el edificio tiene más fuerza a la hora de demarcar un límite entre los que somos vecinos inmediatos y los otros.

Por otro lado, este resultado revela la misma jerarquía que la evaluación subjetiva del barrio expresada en la encuesta: a menor coincidencia se da menor deseo de cambiarse de barrio y a mayor coincidencia entre el mapa cognitivo de los habitantes y la definición institucional hay mayor deseo de cambio de barrio, o menos arraigo (fig. 8)

A primera vista los resultados anteriores parecen sorprendentes: ¿por qué los habitantes cuya percepción de los límites de su barrio coincide con la definición institucional tienen peor evaluación de sus barrios? Una posible explicación es que los dibujos de las personas pudieron haber reflejado los límites percibidos como el espacio social del barrio, es decir, el ámbito físico donde las personas se encuentran con sus vecinos. En una publicación reciente se sugiere que el principal factor explicativo del arraigo en sectores vulnerables es la presencia y calidad de las redes interpersonales, incluso por sobre el tiempo de residencia en el área, la condición de tenencia, edad o si las personas habían o no crecido en el área (Livingston et al., 2008). Desde esta perspectiva se 
podría entender que los habitantes al mapear su barrio estarían aludiendo a sus redes interpersonales $\mathrm{y}$, aquellos que no se limitan a la definición institucional, reflejan mayor diversidad en el tipo y cantidad de redes interpersonales. Ello también explicaría sus mayores niveles de satisfacción.

\section{CONCLUSIONES}

Después de haber revisado una selección de perspectivas para abordar el tema del barrio y algunas dimensiones relevantes para su mejor comprensión, cabe destacar tres aspectos que enmarcan el enfoque, la magnitud y la forma de abordar la presente investigación. El primero dice relación con la importancia del enfoque territorial y espacial que se elige para aproximarse a los problemas de los barrios populares. Al respecto, varios son los investigadores y expertos del área que han reconocido la importancia y necesidad de la perspectiva territorial después de años de políticas de vivienda y hábitat que menospreciaron estos enfoques.

El segundo dice relación con el interés por abordar los problemas del hábitat en múltiples escalas, lo que también está en línea con la gestión territorial actual. Al respecto, un libro editado por el Banco Interamericano de Desarrollo acerca de los asentamientos informales y periferias pobres recientemente, destaca precisamente la necesidad de "centrarse en la escala territorial en la que se manifiestan los problemas y trabajar en su solución en esa escala. Para muchos de los problemas, esta escala no es la del barrio" (Rojas, 2009).

Por último, en cuanto a la búsqueda por una batería de variables que afectan el complejo fenómeno de la consolidación barrial, se busca evitar las respuestas deterministas y se opta por respuestas probabilísticas. Por ejemplo cuando Hillier (1996) analiza comunidades urbanas, describe "campos potenciales de encuentro y copresencia", agregando "más allá, es efecto de la cultura".
Resumiendo las principales tendencias vistas en este estudio de casos, se encontró que a pesar de ser conjuntos habitacionales socioeconómicamente homogéneos y localizados en un mismo contexto urbano, los conjuntos de departamentos son consistentemente peor evaluados que los de casas. Quizás por eso mismo el impacto del PRB en los conjuntos de edificios, en términos de mejorar las relaciones vecinales y el arraigo de los habitantes, es mayor que el que tiene en los conjuntos de casas. Por su parte, la estructura de la malla urbana demostró tener efectos importantes en el control visual de los espacios públicos. Las plazas internas de los conjuntos de casas ofrecen menos visibilidad de exterior a exterior, pero gran control visual y de los accesos desde el interior de las viviendas al espacio público. En el caso de los departamentos, los mayores campos visuales de exterior a exterior se contrastan con una gran disociación desde el espacio interior al exterior. Asimismo se encontraron señales de una relación de mayor satisfacción cuando se estaba cerca de las plazas de los conjuntos.

También es importante señalar que se demostró total consistencia entre la evaluación y la falta de arraigo al conjunto habitacional: el conjunto de casas intervenido es el de mejor evaluación y también el de mayor arraigo. Al contrario de los departamentos no intervenidos donde la evaluación es más baja y con un menor arraigo. El hecho de que el conjunto con mejor evaluación refleje menor coincidencia con los límites del barrio y que el de mayor coincidencia con los límites del barrio exprese un mayor deseo de mudanza, parece indicar que los límites no son un aspecto apreciado o valorado, sino que más bien se siente como una prisión, un ámbito del cual se desea salir.

Así, el presente estudio apunta a relevar la importancia del territorio para dar respuestas locales que deberán trascender la escala del barrio, apuntando a identificar una batería de factores que afectan los procesos de construcción y consolidación de los barrios populares. ARQ
MARGARITA GREENE | Arquitecta, 1973 y Magíster en Sociología, Pontificia Universidad Católica de Chile, 1988; PhD en Arquitectura y Urbanismo, Bartlett School of Architecture at the University College London, 2002. Los temas principales de su labor como investigadora, docente y consultora, realizada entre Inglaterra y Chile, han sido la vivienda social, el proyecto urbano y la modelación espacial. Actualmente ejerce la docencia en la Escuela de Arquitectura y en el programa de Magíster en Proyecto Urbano de la Pontificia Universidad Católica de Chile, donde es profesora titular.

FELIPE LINK | Sociólogo y Magíster en Investigación Social y Desarrollo, Universidad de Concepción, 2003 y Doctor en Arquitectura y Estudios Urbanos, Pontificia Universidad Católica de Chile, 2010. Ha sido investigador responsable y co investigador en diferentes proyectos en el campo de la sociología urbana, particularmente en temas como redes personales en el espacio metropolitano, fragmentación urbana y desigualdades socio-territoriales. Actualmente es profesor asistente del Instituto de Estudios Urbanos y Territoriales UC.
RODRIGO MORA | Arquitecto, Universidad de Chile, 1996; MSc, 2001 y PHD en Estudios Cognitivos y Space Syntax 2009, Bartlett School of Architecture at the University College London, Reino Unido. Sus principales líneas de investigación se vinculan a la navegación y percepción espacial, donde ha sido investigador responsable y co-investigador de proyectos con financiamiento externo e institucional. Actualmente es profesor adjunto de la Escuela de Arquitectura de la Universidad Diego Portales.

CRISTHIAN FIGUEROA | Arquitecto y Magíster en Proyecto Urbano, Pontificia Universidad Católica de Chile, 2011. Actualmente es colaborador e investigador del Laboratorio de Ciudad y Movilidad fadeu en donde se desempeña en diversos proyectos de investigación y diseño urbano que abordan las temáticas de la movilidad urbana, el transporte público y el espacio público. 


\section{REFERENCIAS BIBLIOGRÁFICAS}

APPLEYARD, Donald. "Styles and Methods of Structuring a City”. Environment and Behavior (2): 100-117, junio de 1970 BORJA, Jordi y MUXI, Zaida. El espacio público: ciudad y ciudadanía. Barcelona, Electa, 2003

BRAIN, Isabel, IACCOBELLI, Andrés y SABATINI, Francisco. "Calidad y valor de la vivienda social: un problema de localización y de barrio". Publicación ProUrbana [en línea] 2005, № 2 [fecha de consulta: 26 de diciembre de 2013]. Disponible en: <http://www.anillosintegracionycohesion. cl/Publicaciones/Publicaciones\%20de $\% 20$ los\%20miembros\%20del\%20equipo/ Publicaciones\%20lsabel\%20Brain/Brain Calidad\%20y\%20valor\%20de\%20la\%20 vivienda\%20social.pdf $>$ BOURDIEU, Pierre. "El efecto de lugar". En: BOURDIEU, Pierre y PASSERON, Jean Claude. La miseria del mundo. México, Fondo de Cultura Económica, 1999. p. 119-124.

Espacios urbanos seguros [en línea]. Santiago, Ministerio de Vivienda y Urbanismo, 2003 [fecha de consulta: 26 de diciembre de 2013]. Disponible en: <http://minvu.cl/ opensite_20070417154508.aspx> GRAVANO, Ariel. El barrio en la teoría social hoy. Buenos Aires, Espacio Editorial, 2003. GEHL, Jan. La humanización del espacio urbano. Barcelona, Reverté, 2006. HIDALGO, Rodrigo. “¿Se acabó el suelo en la gran ciudad? Las nuevas periferia metropolitanas de la vivienda social en Santiago de Chile". EURE, 33 (98): 57-75, mayo de 2007.

HIDALGO, M. Carmen y HERNÁNDEZ, Bernardo. "Place Attachment: Conceptua and Empirical Questions". Journal of Environmental Psychology (21): 273-281, septiembre de 2001.

HILLIER, Bill. Space is the Machine: A Configurational Theory of Architecture. Cambridge, Cambridge University Press, 1996.

JACOBS, Jane. The death and life of great American cities: with a new foreword by the author. 24a ed. Nueva York, Modern Library, 1994.
LIVINGSTON, Mark, BAILEY, Nick y KEARNS, Ade. People's Attachment to place - the influence of neighborhood deprivation. York, Joseph Rowntree Foundation, 2008.

KAZTMAN, Rubén. “Seducidos y abandonados. El aislamiento social de los pobres urbanos". Revista de la CEPAL, (75): 171-189, diciembre de 2001. KAZTMAN, Rubén. "La calidad de las relaciones sociales en las grandes ciudades de América Latina: viejos y nuevos determinantes". Revista Pensamiento Iberoamericano (segunda época, 1): 177-205, 2007.

LEFEVBRE, Henri. De lo rural a lo urbano.

Madrid, Península, 1969.

LYNCH, Kevin. La imagen de la ciudad. Barcelona, Editorial Gustavo Gili, 2010. MAYOL, Pierre. "El barrio". En: De Certeau, Michel (ed). La invención de lo cotidiano México, Editorial Universidad Iberoamericana, 2006. p. 5-12. MÁRQUEZ, Francisca. "Santiago: Modernisation, segregation and urban identities in the twenty-first century".
Urbani izziv, 2 (22): 86-97. 2011. MÁRQUEZ, Francisca y PÉREZ, Francisca. Spatial frontiers and neo-communitarian identities in the city: The case of Santiago Chile". Urban Studies Revue, 45 (7): 1461-1483. 2008.

NEWMAN, Oscar. Crime prevention throught urban design. Nueva York, Macmillan Company, 1972 REYES Sonia y FIGUEROA Isabel. "Distribución, superficie y accesibilidad de las áreas verdes en Santiago de Chile". EURE, 36 (109): 89-110. Diciembre de 2010.

RODRÍGUEZ, Alfredo y SUGRANYES, Ana. Los con techo. Un desafío para la política vivienda social Santiago, Ediciones Sur, 2005.

ROJAS, Eduardo. Construir ciudades. Mejoramiento de barrios y calidad de vida urbana [en línea]. Washington DC: Fondo de Cultura Económica, Banco Interamericano de Desarrollo, 2009 [fecha de consulta: 26 de diciembre de 2013]. Disponible en: <http://www.iadb.org/en/ publications/publication-detail, 7101 .
FROM THE HOME TO THE NEIGHBORHOOD

MARGARITA GREENE | PROFESSOR AND CENTRO DE DESARROLLO SUSTENTABLE RESEARCHER, PONTIFICIA UNIVERSIDAD CATÓLICA DE CHILE, CHILE.

FELIPE LINK | PROFESSOR AND CENTRO DE DESARROLLO SUSTENTABLE RESEARCHER, PONTIFICIA UNIVERSIDAD CATÓLICA DE CHILE, CHILE.
KEYWORDS XXX, XXX

RODRIGO MORA | PROFESSOR, UNIVERSIDAD DIEGO PORTALES, CHILE.

CRISTHIAN FIGUEROA | COLLABORATOR AND INVESTIGATOR FOR THE CITY AND MOBILITY LABORATORY FADEU, PONTIFICIA UNIVERSIDAD CATÓLICA DE CHILE, CHILE.

\section{A SOCIAL-SPATIAL PERSPECTIVE: FROM MORE HOUSING TO BETTER NEIGHBORHOODS}

In the last 100 years of history, Chilean public housing policy has been continually adapting to the social, economic, and political circumstances of the country, adopting numerous strategies and solutions according to the moment. This resulted in its passing from a State provision to a subsidiary, from building large housing complexes of finished homes to progressive small scale housing solutions that must be finished by the occupants themselves. It changed from a model based mainly on public funding to one where the private sector is the protagonist. Also, in the last few years both authorities and experts in the field have expressed the need for a new change in the political housing approach. This change would aim to incorporate a "neighborhood perspective" to housing policy, improving both housing and its surroundings.

The rationale behind this latest change seems to have, at least theoretically, its roots in two papers published in the last 10 years: "The New Urban Poor" by Manuel Tironi (written in 2003) and "Those with Roofs" by Alfredo Rodríguez and Ana Sugranyes, published in 2005. Although different in their approach, the first focuses on the social and environmental dimensions of what it currently means to be poor in Chile. The second work focuses on the absence of community in the socia housing complexes built in Chile since the ' 90 s. Both coincide in that the current problems of Chilean cities are no longer associated with basic material or food shortages (such as the lacking shelter or malnutrition) but with issues of social vulnerability or exclusion (Wacquant, 2001).

The previous diagnosis comes at time when, after more than 30 years of housing policy with an emphasis on the production of new solutions, for the first time we are foreseeing overcoming the quantitative housing deficit in the short term (Hidalgo, 2007). This reality has allowed us to rethink the design of traditional housing policies and instruments geared towards the production of housing solutions to the urban environment in which they are installed. Thus, the improvement of public space, of neighborhood relationships and citizen participation acquire more and more relevance in the sectoral discussion. It is no longer just about producing housing, but how both new and existing housing form part of neighborhoods with infrastructure and services that, in turn, are properly inserted in their cities. In summary, the focus moves from a quantitative to qualitative perspective and from a "sectoral" vision -centered strictly on housing- to a more integrated one that includes improving infrastructure and the neighborhood along with strengthening the community.

\section{THE NEIGHBORHOOD}

This new paradigm has led to the emergence of the neighborhood as a unit of urban intervention. The value of the neighborhood as an identifiable territorial unit has been promoted since the middle of last century. By 1960 Lynch emphasized their morphological characteristics, while Jacobs highlighted its ability to permit the exchange of relationships and information for the inhabitants. The concept of a neighborhood for Lefevre (1967) appears as a more accessible meeting poin between the geometric space and the social space, that is, as the element that articulates the social space with the physical space and whose structure largely depends on the general structure of the city.

For Mayol, in his article "The neighborhood" from 2006, this stands on practices such as greeting, walking in a certain way or knowing one another in a neighborly way that over time forges a common identity, building confidence and belonging. Then the neighborhood would be the result of a collective imagination that brings together the ideas of community in its residents, operating like a front yard to the home. The neighborhood is also a place where space is negotiated, where different groups try, sometimes covertly, sometimes more explicitly, to impose their values and lifestyles (Gravano, 2003).

In fact, several writers identify the erosion of community as a result of the new urban poverty. Katzman (2001) suggests that the consolidation process of vulnerable neighborhoods in Santiago appear to limit the potential attributed to the neighborhood scale, often converting it into an isolating element for the urban poor. Thus, this research attempts to advance the understanding of consolidation processes of popular neighborhoods and communities in order to realize the complexity of the neighborhood as a unit of analysis. Thus, this study ${ }^{1}$ is performed through a systemic perspective considering multiple variables that interact both simultaneously and sequentially at more than one urban scale.

THE CASE STUDIES TO BE ANALYZED Beginning with the neighborhoods of the Chilean Ministry of Housing and Urbanism MINVU's "Neighborhood Recovery Program" (PRB) from 2010, the study focuses on two relatively close housing developments: one of 
houses (Santa Elena II) and another of apartments (San Francisco) and two additional ones, one of each type that did not receive PRB interventions (Las Acacias and Vicente Huidobro, respectively). The four cases analyzed are located in the municipality of $\mathrm{El}$ Bosque, on the southern fringes of Santiago between the axes of Santa Rosa and Gran Avenida. Located within a radius of $500 \mathrm{~m}$, all are located in the poorest areas of the capital with few green areas and public spaces and an infrastructure deficit (Reyes y Figueroa, 2010). The logic of this sampling can be schematized; grain and location of the developments can be indicated in a simple manner (Table 1 and fig. 1).

The morphology and building typolog is perhaps the most evident variable (and as such, an almost obvious gateway) when it comes to defining and understanding these types of neighborhoods. In this sense, the selection responded to physical and historical aspects that lend homogeneity to this sector of the city: building height, predominant materiality, grouping system of the buildings and construction data. Lynch argues that the generation of coherent urban images depends, in part, on the existence of uniform groups based on morphological characteristics such as height, typology, architectural style, or by its urban history or structure (Lynch, 1960).

A number of authors suggest that certain typologies or architectural forms can affect the quality of the community. Gehl (2001) argues that the measured distance between houses can influence the degree closeness among neighbors by enabling more frequent contact. For his part, Newman (1972) suggests that the key to the creation of "healthy" communities is in the definition of the borders and limits of the complexes. Hillier, in Space is the Machine, of 1996, posited instead that there are aspects of the urban fabric that, under certain circumstances, may generate sick communities in the sense of restricting the access of strangers and dissociating the places where women, men and children transit. According to Hillier, the form and quantity in which streets and passages are connected in the city have a direct relationship with the quantity of people that move along them, creating the initial conditions for forming relationships among neighbors. Along with the latter, recent housing policy related to urban safety emphasized the importance of a visual relationship between the house and the street as well as the relationship between the street and the location of services and infrastructure (Espacios urbanos seguros, 2003).

\section{THE THREEFOLD}

\section{SOCIO-SPATIAL PERSPECTIVE}

Aware that the construction of what is known as "neighborhood space" is a multi-dimensional phenomenon, the investigation addressed the issue simul- taneously both from a social and spatial perspective integrating tools and methods that complement and facilitate a more complete understanding of the problem. To accomplish this, three approaches were used: an analysis of the spatial visibility of the principal spaces of each one of the complexes, a study of perception and the use of space, and finally, an analysis of the perceived limits of the neighborhood by means of mental mapping.

\section{VISIBILITY ANALYSIS}

The visibility analysis conducted with the computer program, Depthmap, accounts the more or less visible areas of the analyzed system. The software traced an orthogonal grid (in this case one by one meter), over the free space of the complexes and later the relationships of mutual visibility were measured between all the resulting cells. This provided a numerical value for each cell and a color gradient visualization that ranges from areas with greater fields of vision in warm tones to the spaces with less visibility in cooler tones (fig. 2).

The graph, with respect to public spaces, reveals large distances between the four complexes, specifically in relation to local plazas ${ }^{2}$ (fig. 2). The first cases are the "A" plazas of the Las Acacias complex, which correspond to interior plazas accessed by pathways halfway through the block. They appear less visually integrated to the immediate surroundings, meaning that they are barely visible from the other public spaces of the neighborhood. However, the on-site work reflects that the houses around them visually control these plazas. Also, in addition to windows, the homes have doors entering directly into the plazas. In Santa Elena II, the principal public spaces, the "B" plazas, are presented as highly accessible from a visual point of view and the houses also have windows and direct entrances to the plazas (fig. 3).

In the case of the buildings, identified with the "D" and "E" plazas, corresponding to the Vicente Huidobro and San Francisco respectively, the plazas are larger and more visually integrated with the general public. However, by being separated from the apartment buildings, there is no visual control from the interior or entrances into these spaces (Table 2).

\section{IDENTITY AND SATISFACTION}

A second look at these neighborhoods is related with the existence of common habits of the inhabitants, lending itself precisely to the idea of the neighborhood as a defining space between the private space of the home and the generalized urban space of the city. The results of this analysis, taken from a survey of 405 residents of the area on their social characteristics, community rooting, resident satisfaction and patterns of public space use demonstrated obvious differences mainly arising from building type.

For example, in relation to the building characteristics such as acoustic and thermal insulation, lighting, ventilation or dwelling size, it was detected that houses are more favorably evaluated than apartments, regardless of the intervention of public policy. Effectively, while the housing subdivisions averaged a score of 5.4 and 5.3 respectively, the apartments averaged a 4.7 and 4.5 respectively ${ }^{3}$. The following graph demonstrates the variation among attributes and that apartments were consistently evaluated worse than the houses (fig. 4).

Similarly, the assessment of housing as a space to facilitate domestic activities in its interior such as studying, resting, eating, interact, cook, bathe or receive friends, is significantly better in housing complexes than in apartments. Indeed, while $45 \%$ of the apartments of San Francisco and $49 \%$ of the Vicente Huidobro residents claim that their housing does not provide adequate space for social activities, these percentages are lower in housing subdivisions.

Thus, a synthesis of the housing, neighborhood and community evaluation in the four residential complexes can be observed. The highest scores correspond to the dwellings and the worse to the neighborhood and, again, in the residences the houses score higher than apartments. There is also a significantly lower score of nonintervened apartments compared those intervened upon. This trend maintains in practically all areas of the investigations: housing subdivisions maintained higher ranges compared to apartments. Similarly, scores descended from housing to the community and then to the neighborhood. The results followed the same trend when compared to the capacity of the housing, community and neighborhood around the possibility of permitting a satisfying life and if they would move if possible (fig. 5).

An overall consistency was observed between poor evaluations and a desire to move (fig. 6). It is important to remember that the San Francisco and Santa Elena II were subject to large-scale interventions in their public spaces, specifically the access stairs to the apartments, the roofs and plaza infrastructure. The data collected indicates that the dwelling typology, house or apartment, was crucial to the assessment and even more so whether or not government intervention was received.

The latter suggests that the impact of this kind of intervention program is greater in building complexes than in housing subdivisions and that it improves the relationships between neighbors and overall neighborhood satisfaction. These results appear to align with previous studies that people value houses more than apartments

(Brain, Iaccobelli and Sabatini, 2005). Given that the four complexes are practically at equal distances from the center, it is not strange that the housing subdivisions score higher than apartments, even after they have been intervened. On the contrary, the substantial improvement of the San Francisco project regarding satisfaction in the neighborhood, suggests that interventions from the $\mathrm{PQB}-\mathrm{PRB}^{4}$ programs have had an important impact on the quality of life of its residents.

Why did the same not occur with the houses where the subdivision that did not receive intervention increased in value more than the intervened one? One possible explication, worthy of future investigation, is that the intervened neighborhood had greater problems in its constitution, and so the interventions could have helped to improve it and facilitate closer relationships in the neighborhood although not fully accomplishing the objective.

Moreover, within each neighborhood, the effect of the local scale variables was investigated. For example, it was found that despite the poor systematic evaluation by the residents of the Vicente Huidobro buildings, whose apartments where not improved, displayed some differences in the subjective evaluations according to the specific location within the neighborhood and is proximity to the few available public spaces. While the average residents' score of the neighborhood of this project is a 3.9, the score increases to 4.2 if one lived close to a plaza and decreases to 3.4 if the residence is located far away from the public spaces. In the same way, the community score increases from 4.2 to 4.4 if it is closer to plazas and 3.7 if farther away. While these results do not demonstrate large differences in the other neighborhoods analyzed, yet we can infer a degree of influence in the presence of plazas in the value of the neighborhood. According to Borja and Muxi (2003), although one cannot demand that urbanism resolve the series of problems that appear to be of another order, they should at leas not make them worse. Lastly, we wish to emphasize that the overall assessment of housing shows minor differences between the analyzed complexes (fig. 5). In this sense, the step from the house to the neighborhood as an object of public intervention becomes more important for architecture and urbanism.

Thus, the results coincide with those proposed by Bordieu in "The effect of place" in 1999. The process of consolidating neighborhoods, analyzed from a social perspective is associated not only with the characteristics of each community but also with the physical conditions of the dwelling and surroundings as a determinant structure of social relationships, where the social space is somehow generated within the 
III. THE SPATIAL COGNITION AND MENTAL RECOGNITION OF THE NEIGHBORHOOD The third approach used in this work is related to the capacity of the residents to identify the limits of what they consider to be their neighborhood. The majority of these approximations of the neighborhood that address this definition are centered on the presence of the "other" in space, which would generate conflict through the imposition of certain behavioral patterns and values between the groups in a neighborhood dispute (Márquez, 2011; Márquez y Pérez, 2008).

The approach used in this research deviates from the latter theory, not in the sense of trying to unravel the conflict between the different groups occupying the space, but rather how each one establishes the limits of what they consider their neighborhood. Seen this way, the approach relates to the traditional methods as in Lynch's work (1960) on the image Bostonians have of their city. According to this vision, the image of a city originates from the interplay of five elements: nodes, paths, roads, neighborhoods and landmarks, which, when viewed repeatedly, generate what is known as a cognitive map of a city in the short term (Tolman, 1948; Siegel and White, 1975; Thorndyke and Hayes-Roth, 1982).

In the present study, the participants were asked to outline the borders of what they considered as their neighborhood. For this they were given a black and white plan with the principal streets and landmarks with the result of their different cognitive maps (fig. 7). In this first instance, the maps were analyzed by calculating the averages and standard deviation of the area and perimeter of the drawn plans. The results show that of the four analyzed complexes; three have an average area between 499 and 532 ha while the Santa Elena II has an average area of 361 ha. Furthermore, the latter has a smaller standard deviation. That is, the drawings were more similar to each other than those of the other three. Unlike the area, the drawings showing perimeters appear to show more consistency: whereas the housing subdivisions perimeters have average lenghts of 2,043 and 2,524 meters, the apartment buildings reach average values of 2,087 and 2,285 meters (Table 3).

Finally, the study considered the coincidence of the borders drawn by the inhabitants with those institutionally defined by the intervention program and by the municipality. Here some important differences were found: the apartment complexes coincided more than the houses. In the Vicente Huidobro complex the borders coincided $45 \%$, in the San Francisco complex the percentage was $36 \%$, Santa Elena II at $32 \%$ and Las Acacias at $14 \%$. This suggests that at first glance the building complex draws a stronger line between immediate neighbors and the rest.

On the other hand, this result reveals this same hierarchy as the subjective assessment of the neighborhood expressed in the survey: less coincidence is linked with less desire to change neighborhoods, and more coincidence in the cognitive map of the residence and the institutional definition with greater wish to move neighborhoods or less rooting (fig. 8).

At first glance the above results seem surprising: Why do the residents whose perception of neighborhood limits coincide with the institutional definition have lower evaluations of their neighborhoods? One possible explanation is that the people's drawings could have reflected the perceived limits as the social space of the neighborhood, that is, the physical area where people meet with their neighbors. A recent publication suggested that the main factor in successful rooting within social vulnerable groups is the presence and quality of interpersonal networks, even more so than the time spent living in the area, living conditions, age, or if the people grew up in the area or not (Livingston et al., 2008). From this perspective it can be understood that the residents mapping their neighborhood would be alluding to their interpersonal networks and, those that are not limited to the institutional definition, reflect greater diversity in the type and quality of interpersonal relationships. This would also explain the higher levels of satisfaction.

\section{CONCLUSIONS}

After having revised a selection of perspectives addressing the neighborhood issue and its relevant dimensions for better understanding, three aspects stand out that frame its focus, the magnitude and form of undertaking the present investigation. The first is related to the importance of the territorial and spatial perspective chosen for approaching the problems of poorer neighborhoods. In this regards, many of the researchers and experts in the area have recognized the importance and necessity of a territorial perspective after years of housing policy that undervalued this approach.

The second is related to the interest for addressing habitat problems at multiple scales, which is also aligned with current land management. In this regard, a book published by the Inter-American Development Bank on informal settlements and lowincome peripheries recently, highlights precisely the need for "focusing on the spatial scale in which problems manifest themselves and work towards a solution at that scale. For many of these problems, this is not the neighborhood scale" (Rojas, 2009).

Finally, while searching for a set of variable that affects the complex phenomenon of neighborhood consolidation, one must avoid the deterministic responses and opt for probabilistic answers. For example, when Hillier (1996) analyzed urban communities, he describes "potential areas of co-presence and encounter," adding "beyond that, it is the effect of culture."

Summarizing the main tendencies seen in this case study, it was found that despite being socio-economically homogenous housing complexes and located in the same urban context, the apartment buildings are consistently more poorly evaluated than the houses. Perhaps this is why the impact of the PRB in the building complexes, in terms of improving neighborhood relationships and resident settling, is greater than those of the housing subdivisions. Meanwhile, the structure of the urban grid proved to have significant effects on the visual control of public plazas. Internal plazas of the housing complexes offer less visibility from exterior to exterior, but greater visual and access control from the interior of the homes to the public space. In the case of the apartments, the larger field of vision from exterior to exterior is contrasted by a strong dissociation from the interior space to the exterior. As such, greater relationship satisfaction was observed when dwellings were close to the plazas.

It is also important to note that a complete consistency was observed between the evaluation and the lack of rooting in a housing complex: the intervened housing subdivisions received the highest evaluation and index of settling. On the contrary, the non-intervened apartment complexes were the ones where the evaluation was lower and with higher resident turnover. The fact that the complex with the best evaluation reflects less coincidence with the neighborhood limits and that with the highest limits coincidence appears also a higher desire to move seems to indicate that the limits are not an appreciated or valued aspect, but rather, they reinforce a "prison-like feeling", an environment that one wishes to leave.

Thus, the present study aims to highlight the importance of the local territory to give local solutions that transcend the neighborhood scale, seeking to identify a set of factors that affect the processes of construction and consolidation of low-income neighborhoods. ARQ

Notes

1 This investigation had the support of the FONDECYT PROJECT 1100068 Consolidation of vulnerable neighborhoods from a socio-spatial perspective. This also had the support of CEDEUS

-Center for Sustainable Urban Development, Chile project CONICYT/FONDAP 15110020.

2 For effects of the study, the plazas are identified with letters from $\mathrm{A}$ to $\mathrm{E}$.

3 In a scale from 1 to 7 , equivalent to that of the grades at Chilean primary schools. 4 "Programa Quiero mi Barrio"-I love my neighborhood program- and "Programa de Recuperación de Barrios"-Neighborhood recuperation program- respectively.
MARGARITA GREENE | Architect, 1973 and Master in Sociology, Pontificia Universidad Católica de Chile, 1988; PhD in Architecture and Urbanism, Bartlett School of Architecture at the University College London, 2002. The principal themes of her work as researcher, docent, and consultant, realized in England and Chile, have been social housing, the urban project and spatial modeling. She currently teaches in the School of Architecture and in the Master in Urban Projects program at the Pontificia Universidad Católica de Chile, where she acts as professor.

FELIPE LINK | Sociologist and Master in Social Research and Development, Universidad de Concepción, 2003 and Doctor of Architecture and Urban Studies, Pontificia Universidad Católica de Chile, 2010. He has been the main researcher and co-researcher in various projects in the field of urban sociology, particularly on themes such as personal networks in the metropolitan space, urban fragmentation and socio-territorial inequality. He is currently assistant professor at the Instituto de Estudios Urbanos y Territoriales UC.
RODRIGO MORA | Architect, Universidad de Chile, 1996; MSc, 2001 and PhD in Cognitive Studies and Space Syntax 2009, Bartlett School of Architecture at the University College London, United Kingdom. His principal lines of investigation are linked to spatial navigation and perception where he has been chief investigator and co-investigator of projects with external and institutional financing. He is currently an adjunct professor of the Universidad Diego Portales School of Architecture.

CRISTHIAN FIGUEROA | Architect and Master of Urban Project, Pontificia Universidad Católica de Chile, 2011. He is currently a collaborator and researcher of the Laboratorio de Ciudad y Movilidad fadeu where he participates in diverse projects of investigation and urban design that deal with urban mobility, public transport and public space. 